\title{
Efficacy of catheter lock solutions
}

Antimicrobial-containing citrate lock solutions are more effective than heparin lock solutions for the prevention of catheter-related bloodstream infections (CRBSIs), say researchers. In their metaanalysis of 13 randomized controlled trials, Fu Ping and colleagues found that use of citrate lock solutions containing antimicrobials-but not citrate lock solutions alone-significantly reduced the incidence of CRBSIs $(P<0.001)$ compared with use of heparin lock solutions in patients with central venous catheters who were receiving haemodialysis $(n=1,770)$. However, in their subgroup analysis, high-concentration citrate lock solutions were not more effective than heparin lock solutions in preventing CRBSIs.

The researchers also report that the incidences of exit-site infections, catheter removal for poor flow, thrombolytic treatment, all-cause death, catheter thrombosis and catheter-related readmissions, as well as mean catheter durations and CRBSI-free catheter survival did not differ between patients who received citrate locks and those who received heparin locks. However, the incidence of bleeding episodes was significantly lower in the citrate lock group than in the heparin lock group $(P<0.002)$.

"Our study demonstrates that locking central venous catheters with antimicrobial-containing citrate solutions could effectively reduce the incidence of CRBSIs," concludes Fu. "As citrate itself has an antimicrobial effect, the finding that low-to-moderate concentration but not high concentration citrate locks are superior to heparin locks for the prevention of CRBSIs is interesting. Further studies are required to identify the optimal concentration of citrate in lock solution to prevent CRBSIs and the underlying mechanism of protection."

Ellen F. Carney

Original article Zhao, Y. et al. Citrate versus heparin lock for hemodialysis catheters: a systematic review and metaanalysis of randomized controlled trials. Am. J. Kidney Dis. doi:10.1053/j.ajkd.2013.08.016 